\title{
VARIAÇÕES MORFOLÓGICAS DO LÓBULO EM LEJEUNEA GLAUCESCENS GOTT. (LEJEUNEACEAE, HEPATICOPSIDA).
}

\author{
Cid José PASSOS BASTOS e Silvana B. VILAS BÔAS-BASTOS
}

\begin{abstract}
RESUMO. Variações morfológicas do lóbulo em Lejeunea glaucescens Gott. (Lejeuneaceae, Jungermanniophyta). Foram estudados exemplares de Lejeunea glaucescens Gott. no sentido de serem estabelecidos padrões morfológicos objetivando melhor delimitar esse táxon, sendo identificados 10 padrões de variação nos lóbulos dos filídios. Análises desses padrões mostram que não há uma clara correlação com as condições ambientais.
\end{abstract}

Palavras-chave. Lejeuneaceae, Lejeunea glaucescens, morfologia, Bahia.

ABSTRACT. Morphological variations of the lobule in Lejeunea glaucescens Gott. (Lejeuneaceae, Jungermanniophyta). Specimens of Lejeunea glaucescens Gott. were studied in order to identified morphological patterns to the best delimits this species, being recognized 10 morphological patterns in the lobules. Analysis of these show that there are not clear correlation with environmental conditions.

Key words. Bryophyte, Lejeuneaceae, Lejeunea glaucescens, morphology, Bahia.

\section{INTRODUÇÃO}

Variações na morfologia dos gametófitos de representantes das Lejeuneaceae são freqüentes, principalmente em Lejeunea, gênero bastante complexo e que no Brasil conta com 64 espécies, segundo dados de Yano (1996). Schuster (1980) reconhece para esse gênero 05 subgêneros e 10 seções: Subgênero Apolejeunea Inoue, com a Seção Protolejeunea Schust.; Subgênero Lejeunea Libert, com as seções Flavae Schust., Lamacerinae Schust., Lejeunea Schust., Cladiophorae Schust., Glaucescentes Schust., Floridanae Schust., Minutilobae Schust., Cladogynae Schust.,
Microstipulata Schust.; Subgênero Microlejeunea Spruce; Subgênero Nanolejeunea Schust. e Subgênero Crossotolejeunea Spruce.

No entanto, nem todos os subgêneros são reconhecidos por outros autores, que os elevam à categoria de gênero, como Microlejeunea, por exemplo. Estudos mais acurados, auxiliados por novas técnicas em taxonomia são necessários para delimitar melhor os táxons de Lejeuneaceae. Os estudos de variações morfológicas seriam uma ferramenta a mais nessa tentativa, uma vez que variações na morfologia são observadas, muitas vezes, em um mesmo indivíduo. 
De acordo com Schuster (1980), Lejeunea glaucescens Gott. é uma espécie polimórfica, de larga distribuição, ocorrendo em ambientes mésicos, crescendo sobre solos, troncos de árvores e troncos em decomposição. Schuster (op. cit.) reconhece três variedades, $L$. glaucescens Gott. var. glaucescens Schust., L. glaucescens Gott. var. obsoleta Schust. e $L$. glaucescens Gott. var. acrogyna Schust. No entanto, a grande variabilidade morfológica desse táxon torna difícil a delimitação de tais variedades.

Variações morfológicas no gametófito são de ocorrência comum entre as Lejeuneaceae, principalmente em Lejeunea Libert. Essas variações ocorrem, principalmente, no lóbulo e, em menor grau, nos anfigastros, como observadas em Lejeunea brittoniae (Evans) Grolle, Lejeunea laetevirens Nees \& Mont., Lejeunea maxonii (Evans) He e em algumas espécies de Microlejeunea. Podem ser observadas, também, variações no número de células medulares do caulídio em algumas espécies. Lejeunea glaucescens Gott é uma das espécies na qual essas variações são mais acentuadas. Essa espécie apresenta lóbulos bastante variáveis, tanto na forma quanto na dimensão, e em menor grau nos anfigastros.

Lejeunea glaucescens, segundo Schuster (1980), está estreitamente relacionada a Lejeunea calcicola Schust., diferindo desta na forma do lobo e do anfigastro, mais largo do que longo, nesta última. Entretanto, já foram observados anfigastros muito semelhantes aos de Lejeunea calcicola, podendo ser a delimitação entre os dois táxons tarefa difícil.

O objetivo do trabalho é estabelecer os padrões de variação do lóbulo para melhor delimitação desse táxon.

\section{MATERIAL E MÉTODOS}

Foram analisadas 60 amostras de Lejeunea glaucescens Gott. procedentes de localidades dos estados do Acre, Bahia, Rio de Janeiro, Rondônia e São Paulo, depositadas nos seguintes Herbários: Herbário Alexandre Leal Costa (ALCB), do Instituto de Biologia da Universidade Federal da Bahia, Herbário do Centro de Pesquisas do Cacau (CEPEC), Herbário Barbosa Rodrigues (RB), do Jardim Botânico do Rio de Janeiro e Herbário Científico do Estado "Maria Eneyda P. Kauffmann Fidalgo", do Instituto de Botânica da Secretaria de Estado do Meio Ambiente de São Paulo (SP). Também foram examinados exemplares da coleção particular do Briologista Daniel Moreira Vital, da Seção de Briologia e Pteridologia do Instituto de Botânica de São Paulo, gentilmente cedidos para esse trabalho. As identificações foram baseadas em Schuster (1980), ilustrações foram feitas em câmaraclara acoplada ao microscópio Olympus CBA e medidas das estruturas obtidas com ocular micrométrica. Os conceitos de Subgênero e Seção adotados nesse trabalho foram os de Schuster (1980).

\section{TRATAMENTO TAXONÔMICO}

Lejeunea glaucescens Gott., como estabelecido por Schuster (1980), pertence à Seção Glaucescentes do Subgênero Lejeunea, circunscrita com base nos seguintes caracteres: lóbulos dos filídios sempre pequenos, reduzidos a vestigiais, algumas vezes inflados; anfigastros pequenos, a base estreita a arredondada, nunca cordada.

Lejeunea glaucescens Gott. in Gott., Lindenb. \& Nees, Syn. Hep. 378. 1845.

Localidade-tipo: Brasil.

Gametófitos pequenos a medianos, $1 \mathrm{~mm}$ $1,3 \mathrm{~mm}$ de largura, verdes, prostrados, pouco ramificados, as ramificações do tipo-Lejeunea. Caulídio em secção transversal com 7 células corticais e 14-16 células medulares. Filídios 

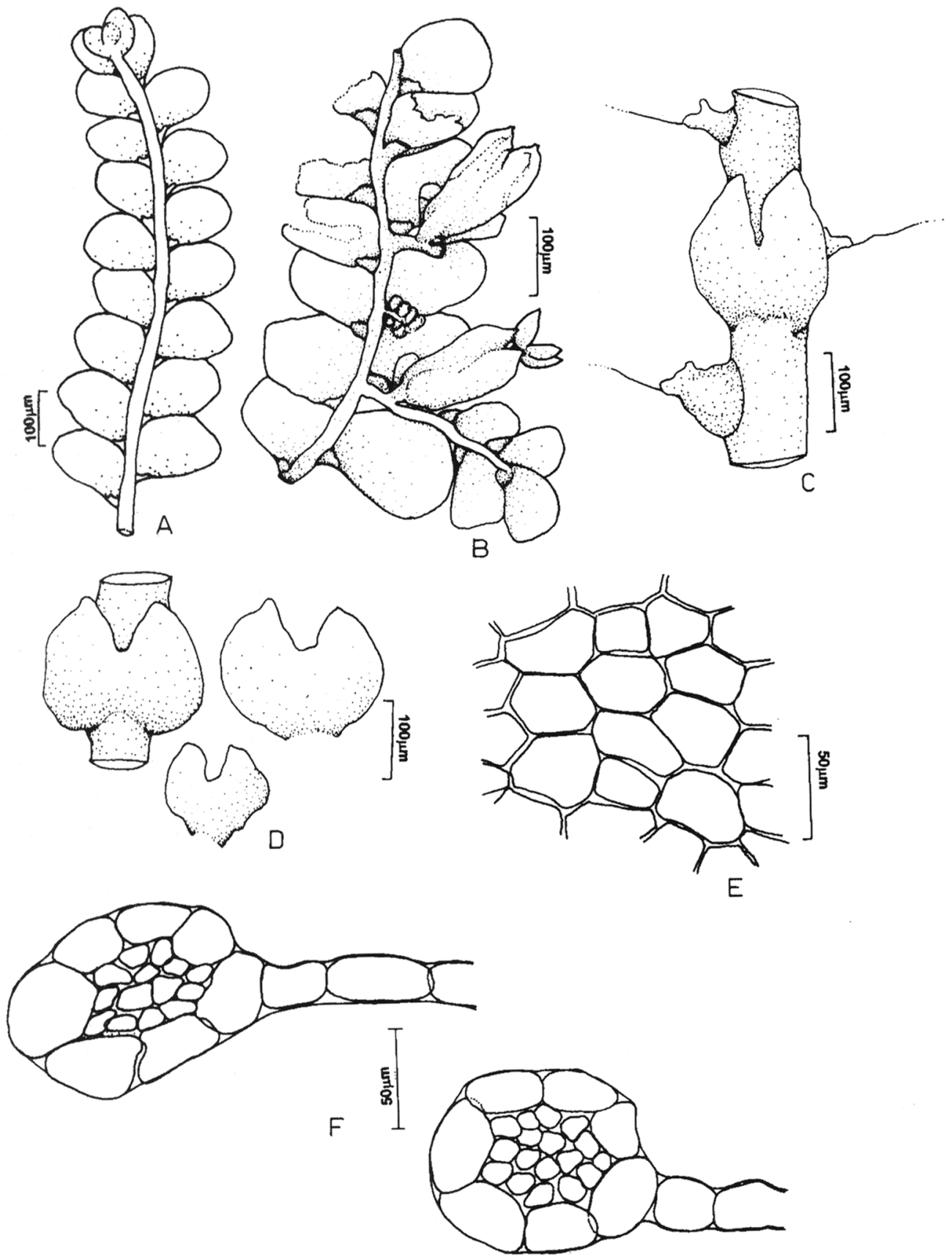

Figura 1. Lejeunea glaucescens Gott. a) gametófito, vista ventral; b) gametófito com ginoécios, vista ventral; c) detalhe de um ramo com lóbulos e anfigastro; d) anfigastros; e) células da lâmina do lobo; f) seções transversais do caulídio (ALCB 38236). Lejeunea glaucescens Gott. a) gametophyte, ventral view; b) gametophyte with gynoecia; c) lobule and underleafy; d) underleaves; e) lamina cells of lobe; f) transversal sections of the stem. 
patentes, contíguos, pouco imbricados; lobo orbicular, $163,4 \mathrm{~mm} \times 118,2 \mathrm{~mm}$, margem lisa; células hexagonais, 40,4mm x 26,2mm-45,4mm x $26,2 \mathrm{~mm}$, paredes delgadas a espessas, trigônios e espessamentos intermediários inconspícuos; oleocorpos pequenos, elipsóides, segmentados; lóbulo inflado ovalado, 93,7mm x $56,2 \mathrm{~mm}-100 \mathrm{~mm} \times 87,5 \mathrm{~mm}$, dente apical curto; lóbulo reduzido curto-retangular a quase plano, 68,7mm X 43,7mm-145,2mm x 66,6mm. Anfigastros pequenos, 131,2mm-168,7mm de largura, orbiculares a ovalados, a base estreita, geralmente não cordada, os lobos eretos a pouco divergentes. Merófito ventral com 2 células de largura. Androécio em curtos ramos laterais, brácteas infladas, em 3-5 séries. Ginoécio em curtos ramos laterais, perianto inflado, oblongo, com 5 quilhas, (fig. 1).

Material examinado: Brasil, Bahia: município de Santo Amaro, 19/8/1976, D.M. Vital 6549 (ALCB 41492); município de São Francisco do Conde, Monte Recôncavo, Fazenda Engenho Madruga, 18/5/1991, C. Bastos 142 (ALCB 18275); 19/5/1991, C. Bastos 165, 204 (ALCB 18295; 18325); município de Jaguaripe, Jacuruna, 29/6/1991, C. Bastos 253 (ALCB 29245); Ilha do Medo, 19/12/1991, C.S.N. Guimarães s.n. (ALCB 18494; 18496); município de São Sebastião do Passé, Lamarão do Passé, 26/10/1998, S.B. Vilas Bôas Bastos 277 (ALCB 38236); município de Alagoinhas, Campus II -UNEB, 13/9/1995, S.B. Vilas Bôas et al. 60, 80, 86, $92,94,97 ; 101,104 ; 107 ; 123 ; 130,132,134$, 135 (ALCB 28085; 28105; 28111; 28117; $28119 ; 28122 ; 28126 ; 28129 ; 28142 ; 28148$; 41487; 41488; 41489, 28536); município de Salvador, zona urbana, Vale de Nazaré, 27/7/ 1990, C. Bastos 69 (ALCB 18739); Parque Zoobotânico, área antrópica, 9/4/1996, C. Bastos 802 (ALCB 28497); Parque Metropolitano de Pituaçu, 2/12/1996, M. Fonseca et al. s.n. (ALCB 29250); 28/10/1997,
C. Bastos 989, 990 (ALCB 32515, 32516); 4/6/ 1999, C. Bastos 1497, 1499, 1508, 1510, 1511 (ALCB 40614; 40627, 40616; 40625, 40628); Campus Universitário-UFBA, Ondina, área antrópica, 14/8/1991, C. Bastos 393 (ALCB 18595); 29/6/1999, C. Bastos et al. 1530 (ALCB 41505); 11/8/1999, C. Bastos 1532 (ALCB 41507); município de Camaçari, Pólo Petroquímico de Camaçari, área antrópica industrial, 18/10/1998, S.B. Vilas Bôas Bastos 172, 181, 182 (ALCB 38132; 38141; 38142); município de Una, Reserva Biológica do Micoleão (IBAMA), 23/8/1994, L.L. Oliveira 05 (ALCB 41671; CEPEC 63961); Estação Ecológica Veracruz, entre Eunápolis e Porto Seguro, Trilha Tropical, 10/6/1999, S.B. Vilas Bôas Bastos \& C. Bastos 354, 426, 428, 445, 446, 460, 476 (ALCB 41538, 41614; 41617, 41634; 41635, 41647; 41666); São Paulo: Mogi Guaçu, 6/2/1977, D.M. Vital 6972 (ALCB 41490), det. R. Grolle; Ilha do Cardoso, 1/6/ 1982, D.M. Vital 10441 (ALCB 41491), det. R. Grolle. Roraima: Jaru, 9/10/1986, D.M. Vital 14272 (ALCB 41494), det. R. Grolle. Acre: Rio Branco, 26/5/1993, D.M. Vital 14852, (ALCB 41493). Rio de Janeiro: Reserva Biológica do Poço das Antas (RB 315168); município de Angra dos Reis, Ilha Grande, trilha para o Pico do Papagaio, entre 50-110m de altitude, 21/3/1995, O. Yano et al. 23560 (SP 281933).

\section{RESULTADOS E DISCUSSÃO}

Entre as amostras analisadas de Lejeunea glaucescens Gott. foi possível a identificação de 10 padrões morfológicos de variação nos lóbulos (fig. 2). Os padrões I e II representam o lóbulo típico, normal, o qual é pequeno, ovalado, inflado. O padrão III está representado por um lóbulo levemente inflado, com margem livre levemente involuta, sem dente apical aparente; o padrão IV por um lóbulo plano, triangular, com dente apical deslocado para o 

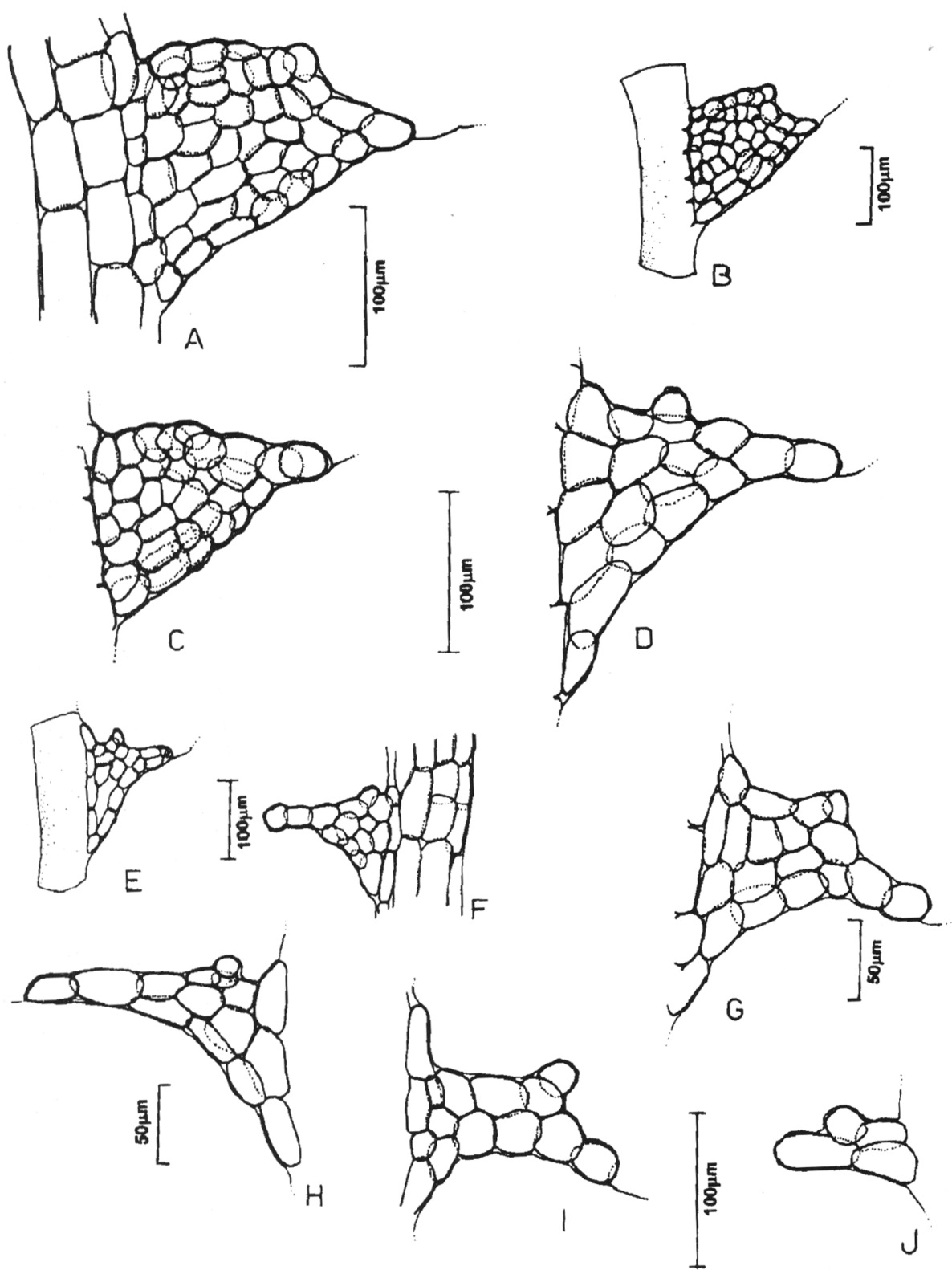

Figura 2. Padrões de variação morfológica do lóbulo de Lejeunea glaucescens Gott. a) padrão I (ALCB 29245); b) padrão II (ALCB 28105); c) padrão III (ALCB 28129); d) padrão IV (ALCB 28129); e) padrão V (ALCB 28105); f) padrão VI (ALCB 28148); g) padrão VII (ALCB 18595); h) padrão VIII (ALCB 18496); i) padrão IX (ALCB 28126); j) padrão X (ALCB 41493). Morphological patterns in the lobule of Lejeunea glaucescens Gott. a) pattern I (ALCB 292545); b) pattern II (ALCB 28105); c) pattern III (ALCB 28129); d) pattern IV (ALCB 28129); e) pattern V (ALCB 28105); f) pattern VI (ALCB 28148); g) pattern VII (ALCB 18595); h) pattern VIII (ALCB 18496); pattern IX (ALCB 28126); j) pattern X (41493). 


\begin{tabular}{|c|c|c|c|c|c|c|c|c|c|c|c|}
\hline AMOSTRAS & I & II & III & IV & $\mathrm{V}$ & VI & VII & VIII & IX & $\mathrm{X}$ & $\mathrm{F}(\%)$ \\
\hline 1 & $x$ & $\mathrm{x}$ & $x$ & & & & & & & & 30 \\
\hline 2 & $\mathrm{x}$ & $\mathrm{x}$ & $x$ & & & & & & & & 30 \\
\hline 3 & $\mathrm{X}$ & $\mathrm{x}$ & $\mathrm{x}$ & $x$ & & & & & & & $\begin{array}{l}40 \\
30\end{array}$ \\
\hline 4 & $x$ & $x$ & $\mathrm{X}$ & $\mathrm{x}$ & & & $x$ & $x$ & & & $\begin{array}{l}30 \\
30\end{array}$ \\
\hline $\begin{array}{l}5 \\
6\end{array}$ & X & $X$ & $\hat{x}$ & $\mathrm{x}$ & & & $\mathrm{x}$ & $x$ & & & 40 \\
\hline 7 & & & $x$ & $\mathrm{x}$ & & & $x$ & & & & 30 \\
\hline 8 & & & $\mathrm{x}$ & $\mathrm{x}$ & & & $x$ & & & & 30 \\
\hline 9 & & & $\mathrm{x}$ & & & & $x$ & & & & 20 \\
\hline 10 & $x$ & & $\mathrm{X}$ & $\mathrm{x}$ & & $\mathrm{x}$ & & & & & $\begin{array}{l}40 \\
30\end{array}$ \\
\hline 11 & $x$ & $x$ & $x$ & & & & & & & & 20 \\
\hline $\begin{array}{l}12 \\
13\end{array}$ & $x$ & $x$ & $\mathrm{X}$ & & $x$ & & $x$ & & & & 30 \\
\hline 14 & & & $\mathrm{x}$ & $\mathrm{x}$ & & & & & & & 20 \\
\hline 15 & $x$ & & & $\mathrm{x}$ & & & & $x$ & & & $\begin{array}{l}30 \\
30\end{array}$ \\
\hline 16 & $\mathrm{x}$ & $\mathrm{x}$ & & & & & & $x$ & & & $\begin{array}{l}30 \\
20\end{array}$ \\
\hline 17 & & & & $\begin{array}{l}x \\
x\end{array}$ & $x$ & $x$ & $\mathrm{X}$ & & & & $\begin{array}{l}20 \\
50\end{array}$ \\
\hline $\begin{array}{l}18 \\
19\end{array}$ & $\begin{array}{l}X \\
x\end{array}$ & $x$ & $x$ & $\mathrm{x}$ & & & $\mathrm{x}$ & & & & 30 \\
\hline $\begin{array}{l}19 \\
20\end{array}$ & & & & & & & & & & $\mathrm{x}$ & $\begin{array}{l}10 \\
30\end{array}$ \\
\hline 21 & & $x$ & & & $\mathrm{X}$ & & & $\mathrm{X}$ & & & $\begin{array}{l}30 \\
40\end{array}$ \\
\hline 22 & $\begin{array}{l}\mathrm{X} \\
\mathrm{x}\end{array}$ & & & $x$ & & & $x$ & $x$ & & & $\begin{array}{l}40 \\
30\end{array}$ \\
\hline 23 & X & $x$ & & $x$ & & & $\mathrm{x}$ & & & & 30 \\
\hline $\begin{array}{l}24 \\
25\end{array}$ & $\mathrm{x}$ & $\begin{array}{l}X \\
x\end{array}$ & $x$ & $x$ & & & $x$ & $\mathrm{X}$ & & & 40 \\
\hline $\begin{array}{l}25 \\
26\end{array}$ & & & $x$ & & & & $x$ & & & & 20 \\
\hline 27 & $\mathrm{x}$ & & & $x$ & & & $x$ & & & & 30 \\
\hline 28 & $\mathrm{X}$ & $x$ & $\mathrm{x}$ & & & & & & $x$ & $x$ & $\begin{array}{l}30 \\
60\end{array}$ \\
\hline 29 & $\mathrm{x}$ & & $x$ & & $x$ & & $\mathrm{X}$ & & $\mathrm{x}$ & & 30 \\
\hline 30 & $\mathrm{x}$ & $\mathrm{X}$ & $\mathrm{x}$ & & & & $x$ & & & $x$ & 50 \\
\hline 31 & $\mathrm{x}$ & $\begin{array}{l}x \\
x\end{array}$ & $\begin{array}{l}x \\
x\end{array}$ & & & $\mathrm{x}$ & $\lambda$ & $x$ & & $x$ & 60 \\
\hline 32 & $\begin{array}{l}x \\
x\end{array}$ & $\begin{array}{l}\mathrm{X} \\
\mathrm{x}\end{array}$ & $\begin{array}{l}x \\
x\end{array}$ & & & & $x$ & & & & 40 \\
\hline $\begin{array}{l}35 \\
34\end{array}$ & $\hat{x}$ & $\mathrm{x}$ & $\mathrm{x}$ & & & & $\mathrm{x}$ & $x$ & & $x$ & 60 \\
\hline 35 & $\mathrm{x}$ & $x$ & $x$ & & & & & & & & 30 \\
\hline 36 & $\mathrm{x}$ & & & & & & $\mathrm{x}$ & $\mathrm{x}$ & & & $\begin{array}{l}30 \\
30\end{array}$ \\
\hline 37 & $\mathrm{x}$ & $x$ & $x$ & & & & & & & & 30 \\
\hline $\begin{array}{l}38 \\
39\end{array}$ & $\begin{array}{l}x \\
x\end{array}$ & $x$ & $x$ & $\mathrm{x}$ & & $x$ & $x$ & $x$ & & & 40 \\
\hline 40 & $\hat{x}$ & $x$ & $x$ & $\mathrm{x}$ & & & & & & & $\begin{array}{l}40 \\
30\end{array}$ \\
\hline 41 & & & $x$ & $x$ & & & $\mathrm{x}$ & & & & $\begin{array}{l}30 \\
50\end{array}$ \\
\hline 42 & $\mathrm{x}$ & & $x$ & & & & $\mathrm{X}$ & $\mathrm{x}$ & & $\mathrm{X}$ & $\begin{array}{l}50 \\
30\end{array}$ \\
\hline 43 & $\mathrm{x}$ & & $x$ & & & & $x$ & & & & $\begin{array}{l}30 \\
30\end{array}$ \\
\hline $\begin{array}{l}44 \\
45\end{array}$ & $x$ & $x$ & $x$ & & & & & $\mathrm{x}$ & & $x$ & 20 \\
\hline 46 & $\mathrm{x}$ & & $x$ & $x$ & $x$ & & $x$ & & & & 50 \\
\hline 47 & $\mathrm{x}$ & & $x$ & $\mathrm{x}$ & & & & $x$ & & & $\begin{array}{l}40 \\
50\end{array}$ \\
\hline 48 & $\mathrm{x}$ & & $\mathrm{x}$ & $x$ & $x$ & & $x$ & & & & $\begin{array}{l}50 \\
40\end{array}$ \\
\hline 49 & $\mathrm{x}$ & $x$ & $\mathrm{x}$ & & & & $x$ & & & & $\begin{array}{l}40 \\
50\end{array}$ \\
\hline 50 & $x$ & $x$ & $x$ & $\mathrm{x}$ & & & $\mathrm{x}$ & & & $x$ & $\begin{array}{l}50 \\
60\end{array}$ \\
\hline 51 & $\mathrm{x}$ & $\mathrm{x}$ & $x$ & & & & $\mathrm{x}$ & & $\begin{array}{l}\mathrm{X} \\
\mathrm{X}\end{array}$ & $\lambda$ & $\begin{array}{l}00 \\
70\end{array}$ \\
\hline 52 & $x$ & $\mathrm{x}$ & $x$ & & & $x$ & & $\begin{array}{l}x \\
x\end{array}$ & $\hat{x}$ & $x$ & 80 \\
\hline $\begin{array}{l}53 \\
54\end{array}$ & & $\mathrm{X}$ & $\begin{array}{l}x \\
x\end{array}$ & $x$ & $\begin{array}{l}x \\
x\end{array}$ & & $\begin{array}{l}\mathrm{X} \\
\mathrm{X}\end{array}$ & $\begin{array}{l}x \\
x\end{array}$ & & & 60 \\
\hline $\begin{array}{l}54 \\
55\end{array}$ & $\begin{array}{l}\mathrm{x} \\
\mathrm{x}\end{array}$ & $\begin{array}{l}x \\
x\end{array}$ & $\begin{array}{l}\mathrm{x} \\
\mathrm{x}\end{array}$ & $\mathrm{x}$ & X & $x$ & $\hat{\mathrm{X}}$ & & & $x$ & 70 \\
\hline 56 & $x$ & $x$ & $x$ & & & & & & & & $\begin{array}{l}30 \\
40\end{array}$ \\
\hline 57 & $x$ & & $x$ & $x$ & & & $\mathrm{X}$ & & & & $\begin{array}{l}40 \\
50\end{array}$ \\
\hline 58 & $\mathrm{x}$ & & $x$ & $x$ & & & $\mathrm{x}$ & $x$ & & & $\begin{array}{l}50 \\
40\end{array}$ \\
\hline $\begin{array}{l}59 \\
60\end{array}$ & $\begin{array}{l}\mathrm{X} \\
\mathrm{X}\end{array}$ & $\begin{array}{l}x \\
x\end{array}$ & $\begin{array}{l}X \\
X\end{array}$ & $\mathrm{x}$ & & & & $x$ & & & $\begin{array}{l}40 \\
40\end{array}$ \\
\hline TOTAL & 45 & 32 & 45 & 26 & 7 & 7 & 30 & 20 & 5 & 10 & - \\
\hline
\end{tabular}

Tabela 1. Ocorrência e percentuais dos padrões morfológicos de variação do lóbulo em Lejeunea glaucescens Gott. Occurrence and percentage of morphological patterns in the lobule of Lejeunea glaucescens Gott. 
centro da margem livre; o padrão V por um lóbulo reduzido, triangular, com dente apical aparente; o padrão VI por um lóbulo reduzido, plano, dente apical inconspícuo; o padrão VII por um lóbulo reduzido, curto-retangular, com dente apical aparente; o padrão VIII por um lóbulo reduzido, plano, alongado horizontalmente, com dente apical deslocado para a região proximal ao caulídio; padrão IX por um lóbulo reduzido, retangular-alongado, com dente apical proeminente, e o padrão $\mathrm{X}$ por um lóbulo vestigial, formado por 4-6 células, com dente apical pouco conspícuo. Desses padrões, os mais freqüentes foram os padrões I $(75 \%)$, III $(75 \%)$, II $(53,33 \%)$, VII $(50 \%)$, IV $(43,33 \%)$, e VIII $(33,33 \%)$. Os menos freqüentes foram os padrões IX $(8,33 \%), \mathrm{V}$ $(11,66 \%)$, VI $(11,66 \%)$ e X $(16,66 \%)$. Esses valores referem-se à freqüência de ocorrência em 60 amostras analisadas, em que esses padrões se repetem em uma mesma amostra. Assim, foi determinada a freqüência da variabilidade dos padrões por amostra analisada (Tabela 1), o que permite uma visualização da variação morfológica do lóbulo. Os padrões I, II, III, IV e VII são os que mais freqüentemente se combinam entre si e, às vezes, com outros padrões, tendo ocorrido em 59 amostras das 60 analisadas. Dentre esses cinco padrões, o menor número de combinações entre si ocorreu em áreas antrópicas, tendo predominado nessas o padrão VII.

Schuster (1980) considera duas variedades para Lejeunea glaucescens: L. glaucescens var. obsoleta e L. glaucescens var. acrogyna, baseado, na morfologia do lóbulo e na posição do ginoécio. Em relação à morfologia do lóbulo, esse caráter não parece ser útil para delimitar variedades taxonômicas devido às variações morfológicas encontradas, não sendo, portanto, um critério suficientemente claro e definido para delimitação de categorias taxonômicas.

Schuster (1980) refere que lóbulos reduzidos e vestigiais em $L$. glaucescens estão associados com o crescimento em habitats úmidos e sombreados. Porém, não foi observada nenhuma correlação com o ambiente, substrato e local de coleta, pois espécimes coletados em ambientes e/ou localidades diferentes apresentaram nos lóbulos os mesmos padrões de variação, indistintamente (Tabela 2). Como observado na Tabela 2, os padrões mais freqüentes na maioria dos ambientes foram I, II e III, sendo que no Cerrado ocorreram todos os padrões; o padrão X ocorreu em áreas antrópicas, Cerrado e floresta ombrófila; o padrão VI ocorreu em áreas antrópicas, Cerrado e floresta secundária. É provável que as condições ambientais possam, de certa forma, influenciar a ocorrência de padrões morfológicos diferentes em uma mesma planta, mas, no caso em estudo, não determinam essas variações. Assim, por exemplo, o exemplar examinado proveniente de uma área de mata no estado do Acre (ALCB 41493), crescendo sobre o solo, apresentou apenas lóbulos reduzidos a vestigiais, enquanto que, na Bahia, Rio de Janeiro e São Paulo, em áreas de mata, foram encontrados espécimens com outros padrões morfológicos, incluindo lóbulos reduzidos a vestigiais.

Em relação ao substrato, Lejeunea glaucescens é uma espécie euritópica, crescendo sobre solos, material vegetal em decomposição ou como epífita, não se observando, também, nenhuma relação direta com o tipo de substrato. O que determinaria a variação morfológica nos lóbulos dessa e de outras espécies? Essa é uma questão difícil, senão impossível de responder com precisão. Variações fenotípicas são comuns em plantas, muitas vezes influenciadas ou mesmo determinadas por condições ambientais específicas. No caso de Lejeunea glaucescens, bem como de outros membros das Lejeuneaceae, essas variações ocorrem independente do ambiente ou do substrato onde ocorrem, muitas vezes em um mesmo ramo, admitindo-se, portanto, que fatores macro ou microclimáticos e físico-químicos do ambiente 


\begin{tabular}{lcccccc}
\hline \multicolumn{7}{c}{ Ambientes } \\
\hline Padrões & FO & Ce & FS & AA & ZU & MG \\
\hline I & $\mathrm{x}$ & $\mathrm{x}$ & $\mathrm{x}$ & $\mathrm{x}$ & $\mathrm{x}$ & $\mathrm{x}$ \\
II & $\mathrm{x}$ & $\mathrm{x}$ & $\mathrm{x}$ & $\mathrm{x}$ & $\mathrm{x}$ & \\
III & $\mathrm{x}$ & $\mathrm{x}$ & $\mathrm{x}$ & $\mathrm{x}$ & $\mathrm{x}$ & $\mathrm{x}$ \\
IV & $\mathrm{x}$ & $\mathrm{x}$ & $\mathrm{x}$ & & & \\
& & $\mathrm{x}$ & & & & \\
VI & & $\mathrm{x}$ & $\mathrm{x}$ & $\mathrm{x}$ & & \\
VII & $\mathrm{x}$ & $\mathrm{x}$ & $\mathrm{x}$ & $\mathrm{x}$ & & \\
VIII & $\mathrm{x}$ & $\mathrm{x}$ & $\mathrm{x}$ & & & \\
IX & & $\mathrm{x}$ & & & & \\
X & $\mathrm{x}$ & $\mathrm{x}$ & & $\mathrm{x}$ & & \\
\hline
\end{tabular}

$\mathrm{FO}=$ Floresta Ombrófila $; \mathrm{Ce}=$ Cerrado e/ou Tabuleiro; FS = Floresta Secundária; $\mathrm{AA}=$ Área Antrópica; $\mathrm{ZU}=$ Zona Urbana; $\mathrm{MG}=$ Mata de Galeria

Tabela 2. Ocorrência dos padrões morfológicos de variação nos lóbulos de Lejeunea glaucescens nos diversos ambientes. Occurrence of morphological patterns in the lobule of Lejeunea glaucescens in the ecosystems.

não são decisivos para determinar essas variações. É evidente que um fenótipo é determinado por um conjunto de fatores intrínsecos e extrínsecos, mas é difícil definir qual ou quais desses fatores determinam ou influenciam os padrões de variação observados. Testes e experimentações de cultivo em condições controladas e bem definidas, se isso for possível, talvez possam oferecer uma luz a essa questão.

A partir da análise dos exemplares estudados, pode ser verificado que não é seguro diferençar morfotipos ou variedades com base nas variações observadas nos lóbulos, pois elas ocorrem, quase sempre, em um mesmo indivíduo e, muitas vezes, no mesmo ramo, no entanto, a ocorrência dos padrões I, II, III, IV e VII, combinados entre si numa amostra, ainda que em ramos distintos, permite a identificação de um exemplar como sendo Lejeunea glaucescens, principalmente em material estéril.

AGRADECIMENTOS. Os autores são gratos ao Briologista Daniel M. Vital, do Instituto de Botânica de São Paulo pela doação de exemplares de sua coleção particular, e aos curadores dos seguintes Herbários: Herbário Barbosa Rodrigues do Jardim Botânico do Rio de Janeiro (RB) e Herbário do Centro de Pesquisas do Cacau (CEPEC) pelo empréstimo de material, como também do Herbário Científico do Estado Maria Eneyda P. Kauffmann Fidalgo, do Instituto de Botânica de São Paulo (SP) por ter permitido o exame dos espécimes.

\section{LITERATURA CITADA}

SCHUSTER, R. M. -1980- The Hepaticae and Anthocerotae of North America east of the Hundredth Meridian. New York: Columbia Univ. Press, vol. 4, 1334p.il.

YANO, O. -1996- A Checklist of the Brazilian Bryophytes. Boletim do Instituto de Botânica 10: 47-232.

Aceptado para su publicación en marzo de 2000

Endereço dos autores. Universidade Federal da Bahia, Instituto de Biologia, Departamento de Botânica, Campus de Ondina, 40170-280 Salvador, Bahia, Brasil, E-mail: cjpbasto@ufba.br 